\title{
Influencia del control de gestión al valor público generado en servicios dependientes del ministerio de economía, fomento y turismo, Chile
}

\author{
Segundo R. Cabana*, Felicindo H. Cortés, Franco A. Contreras y Valentina F. Vargas \\ Universidad de La Serena, Facultad de Ingeniería, Dpto. de Ing. Industrial, Casilla 554, La Serena-Chile \\ (correo-e: rcabana@userena.cl,fcortes@userena.cl,contrerassambra.f@gmail.com, vargascastrov@gmail.com)
}

* Autor a quien debe ser dirigida la correspondencia

Recibido Jul. 18, 2019; Aceptado Sep. 15, 2019; Versión final Nov. 7, 2019, Publicado Abr. 2020

\begin{abstract}
Resumen
Esta investigación analiza la influencia del control de gestión en el valor público que se genera en los servicios dependientes del Ministerio de Economía, Fomento y Turismo de Chile. Se aplicaron encuestas con tamaño muestral de 375 funcionarios, empleando escala politómica tipo Likert. Los resultados se analizan mediante método de ecuaciones estructurales basado en los mínimos cuadrados parciales. La creación de valor público se explica en un $32,5 \%$ por la relación directa con el logro de resultados desafiantes, que a la vez depende significativamente del desarrollo de competencias, dirección centrada en el control de gestión, asignación de recompensas y una cultura de mejora continua. Así la gestión sistémica de estas variables promoverá la creación de valor público, donde el control de gestión permitirá a los decisores públicos conocer y aprender de forma proactiva, según lo que suceda en cada momento, en los procesos considerados críticos para la organización.
\end{abstract}

Palabras claves: control de gestión; ecuaciones estructurales; valor público; mínimos cuadrados parciales; mejora continua

\section{Influence of management control on public value generated in services dependent on the ministry of economy, development and tourism, Chile}

\begin{abstract}
This research analyzes the influence of management control on the public value that is generated in the services dependent on the Ministry of Economy, Development and Tourism of Chile. Surveys with a sample size of 375 officials were applied, using a Likert-type polytomous scale. The results are analyzed by the structural equations method based on partial least squares. The creation of public value is explained in $32.5 \%$ by the direct relationship with the achievement of challenging results, which, at the same time, depends significantly on the development of competencies, management focused on the management control, allocation of rewards and a culture of continuous improvement. Thus, the systemic management of these variables will promote the creation of public value, where management control will allow public decision makers to know and proactively learn, according to what is happening at all times, in the processes considered critical for the organization.
\end{abstract}

Keywords: management control; structural equations; public value; partial least squares; continuous improvement 


\section{INTRODUCCIÓN}

Hoy más que nunca se le da relevancia al control de las instituciones públicas, porque es fundamental que funcione bien para facilitar la efectividad del gobierno institucional. Los controles se implantan con el fin de detectar, en el plazo deseado, cualquier desviación respecto a los objetivos establecidos por la entidad y de limitar las sorpresas. Los controles fomentan la eficiencia, eficacia y economía de los recursos, reducen el riesgo de pérdida de valor de los activos y ayudan a garantizar la fiabilidad de los estados financieros y el cumplimiento de las leyes y normas vigentes, por tanto, deben tener el apoyo al más alto nivel institucional (Peña, 2014).

Evaluar una organización o unidad de negocio es muy complejo, como dice Barney (2011) y Chakravarthy (1986), el uso de múltiples perspectivas y múltiples medidas de desempeño de una organización genera resultados más confiables y eficientes de la situación actual, Kaplan y Norton avanzan en un marco de conceptualización, rendimiento y medición -Balance Score Card (BSC)- qué al notar la importancia de las perspectivas, incorpora a la evaluación varias dimensiones de desempeño (Arboleda y Arias, 2016). Los mandatarios y los empleados subordinados han reconocido su responsabilidad por establecer, mantener, perfeccionar y evaluar el sistema de control interno de la entidad pública, así como promover las disposiciones necesarias para garantizar razonablemente una alta eficiencia en su implantación y funcionamiento, con el fin de estimular la eficiencia de las operaciones, mediante la aplicación de instrumentos idóneos de gerencia que fortalezcan el cumplimiento íntegro y oportunamente de su misión (Gamboa et al., 2016).

Las capacidades, habilidades, necesidades y cualidades son características individuales que interactúan con la naturaleza del trabajo y de la organización para producir comportamientos, los cuales, a su vez, afectan los resultados. Como ejemplos de criterios para medir el desempeño de los empleados, tenemos: cantidad de trabajo, calidad de trabajo, cooperación, responsabilidad, conocimiento del trabajo, asistencia, necesidad de supervisión, etc. El desempeño refleja principalmente los objetivos de la eficiencia, esto es, alcanzar las metas al tiempo que se utilizan los recursos eficientemente (Chiang y San Martín, 2015).

En este contexto se establece un modelo causal, que da forma a una propuesta cuyo objetivo es analizar el uso efectivo del Cuadro de Mando Integral (CMI), como sistema de control de gestión en los servicios dependientes del Ministerio de Economía, Fomento y Turismo, a través de un diagnóstico estratégico del actual Sistema de Control de Gestión del Ministerio, tal que, dicho modelo causal explique la creación de valor público con base al uso efectivo del CMI, considerando para ello 10 hipótesis. H1: Relación del Liderazgo Centrado en Resultados y su influencia en una Dirección Basada en el Control de Gestión; H2: Contribución del Liderazgo Centrado en Resultados y una Dirección Basada en el Control de Gestión en la instauración de una Cultura de Mejora Continua en la Organización; H3: Dirección Basada en el Control de Gestión y su relación con la Asignación de Recompensas; H4: Dirección Basada en el Control de Gestión y la Cultura de Mejora Continua; H5: Relación entre la Asignación de Recompensas y el Logro de Resultados Desafiantes; H6: Dirección Basada en el Control de Gestión y su relación con el Desarrollo de Competencias; H7: Dirección Basada en el Control de Gestión y su contribución al Logro de Resultados Desafiantes; H8: Incidencia de una Cultura de Mejora Continua en el Cumplimiento de Resultados Desafiantes; H9: Incidencia del Desarrollo de Competencias en el Cumplimiento de Resultados Desafiantes en la organización y H10: Incidencia del Logro de Resultados Desafiantes en la Creación de Valor Público.

\section{OTROS ANTECEDENTES}

Se presentan los principales conceptos a considerar en el estudio y como evidenciar el avance conceptual para establecer las diferentes hipótesis formuladas.

\section{Control de Gestión}

Gran cantidad de estudios analizan el impacto de los sistemas de control de gestión en el desempeño de las organizaciones, estableciéndose como un conjunto de procesos que la empresa emplea para certificar las tareas que se realizan y están encaminadas al logro de sus objetivos, que deben estar establecidos por los representantes de la administración, la gestión de control no se limita a comprobar que las tareas realizadas o las decisiones tomadas han sido correctas, sino que parte de su deber es orientar e influir el comportamiento de la organización, para que así sean alcanzados los objetivos propuestos (Porporato y García, 2011).

\section{Gestión Pública}

La Gestión pública es el conjunto de acciones mediante las cuales las entidades tienden al logro de sus fines, objetivos y metas, que se enmarca en políticas gubernamentales establecidas por el Poder Ejecutivo, se ocupa de la utilización de los medios adecuados para alcanzar un fin colectivo, utilizando mecanismos de decisión para la asignación y distribución de los recursos públicos, y de la coordinación y estímulo de los 
agentes públicos para lograr objetivos colectivos (Tello et al., 2009). Por lo tanto, el desempeño del Estado depende de la calidad y eficiencia de las políticas y el desempeño de la gestión pública. Según Aguilar (2015), la gestión pública es un conjunto de operaciones necesarias para el Estado debido a su rol institucional y modo de gobernación, que permite anticipar, neutralizar o disminuir los riesgos de fallas organizativas, favoreciendo la existencia de una organización publica eficiente y responsable.

\section{Cuadro de Mando Integral}

El Cuadro de Mando Integral (CMI), constituye un marco o estructura creada para integrar indicadores que se deriven de la "estrategia". Para sus creadores Kaplan y Norton (2004), es mucho más que un sistema de medición. Su verdadero poder concurre cuando se transforma de un sistema de indicador a uno de gestión. Sin embargo, consideramos, que esencialmente, el CMI es una herramienta integral y sistémica que se ha convertido en esencial para el control moderno, pero que no se traduce en un sistema de gestión por sí misma, sino que forma parte de un sistema mayor y, solo así, complementa indicadores de medición de resultados con otros que influyen en la generación de determinados impactos (Rivero y Galarza, 2017).

\section{Cuadro de Mando Integral para el Sector Público}

Las entidades no lucrativas y las administraciones públicas, deben considerar la colocación de un objetivo muy amplio en la parte superior de su cuadro de mando integral como representación de su objetivo a largo plazo, por ejemplo: la disminución de la pobreza y el analfabetismo o la mejora del medio ambiente. Los objetivos dentro del cuadro de mando integral se pueden orientar entonces hacia la mejora de un objetivo de alto nivel. En una empresa del sector privado, los indicadores financieros proporcionan la responsabilidad ante los propietarios y los accionistas. En una entidad no lucrativa o una administración pública, sin embargo, los objetivos financieros no son los indicadores que revelan si la agencia cumple con su misión (Kaplan y Norton, 2004).

\section{Contribución del Liderazgo Centrado en Resultados}

El líder transaccional es aquel que fomenta el intercambio de recompensas por parte del directivo y el cumplimiento de las tareas asignadas a los empleados que están a su cargo, apuntando, principalmente, a las necesidades extrínsecas (materiales) de las personas. Este estilo de liderazgo se basa en la clara definición del rol del subordinado y las consecuencias positivas o negativas que implica el cumplimiento o no cumplimiento de los objetivos y metas. El líder transaccional se cuadra rígidamente con las metas organizacionales y se preocupa de que los equipos de trabajo bajo su responsabilidad se ocupen meramente de realizar las acciones pertinentes para alcanzar dichas metas (Varela, 2012).

El control de gestión hace operacional la planeación estratégica, mientras la planeación estratégica tiene qué ver con las grandes líneas de desarrollo de la organización a mediano y largo plazo, para determinar directrices y políticas de gestión en horizontes de entre 3 y 5 años, el control de gestión se concentra en el corto plazo, sobre todo en los períodos anuales para definir los programas, los presupuestos y monitorizar el desempeño y el logro de los objetivos intermedios (Anthony y Govindarajan, 2007).La mejora continua es una de las herramientas básicas para aumentar la competitividad en las organizaciones. Esta filosofía se apoya en la explotación de los recursos de la compañía, especialmente los recursos humanos (Prado, 1998) y en el aprendizaje interno (Schroeder et al., 2002). La mejora continua debe significar un modo de vida dentro de la organización (Bond, 1999), es precisamente esto lo que hace a la mejora continua una herramienta tan valiosa y, a la vez, difícil de implementar hasta sus últimas consecuencias (Marín-García et al., 2014).

\section{Liderazgo Centrado en Resultados y Dirección Basada en el Control de Gestión}

Robbins y Judge (2015) define al líder transaccional como "aquellos que guían o motivan a sus seguidores en búsqueda de metas establecidas al aclarar los requisitos del rol y de la tarea", estableciendo que el liderazgo se centra en primer lugar en los resultados y luego en la manera de cómo alcanzarlos. En cuanto a las empresas que pretendan alcanzar un eficiente funcionamiento, deben asegurar que las personas que se encuentren en el manejo directivo dentro de una organización, implementen las estrategias formuladas alineando los objetivos, formulación estratégica, planificación estratégica, decisiones estratégicas, control estratégico global para que contribuyan a los objetivos organizacionales (Toro et al., 2017).

Ulrich y Smallwood (2007), establecen una relación directa entre el tipo de liderazgo y como ésta influye en una dirección que se basa en el control de gestión, donde el liderazgo centrado en resultados permite a la dirección de la empresa poseer una visión global del riesgo y accionar los planes para su correcta gestión y administración, a través de un análisis general de los procesos implementados dentro de la organización en cada una de las unidades de negocio tanto en el sector público como en el privado, teniendo en cuenta que la existencia de riesgos son inherentes a la ejecución del plan institucional, cumplimiento de los objetivos institucionales y desarrollo del objeto social de la entidad. 


\section{Dirección Basada en el Control de Gestión y el Desarrollo de Competencias}

La ya clásica reflexión de Hayek sobre la dependencia del rendimiento de una organización de la ubicación del poder de decisión al lado del conocimiento relevante, constituye la base para el diseño de una arquitectura organizativa que propicie esta concurrencia - poder de decisión y conocimiento-, y que simultáneamente disponga de mecanismos para ejercer el control sobre la actuación de las personas a las que se les han asignado esos derechos de decisión (Prieto y Santidrián, 2016). Las exigencias por la competitividad son la punta de lanza para que las organizaciones mantengan un alto nivel de especialización de su personal, esta es la razón por la cual la capacitación es una de las formas más efectivas de enfrentar el cambio y de modificar algunas actitudes. El desarrollo de recursos humanos tiene carácter estratégico, consiste no solo en capacitación y desarrollo, sino también en actividades individuales de planificación y desarrollo profesional, desarrollo de la organización y evaluación del desempeño, mediante la capitación los colaboradores adquieren o desarrollan conocimientos y habilidades específicas relativas al trabajo, modificando sus actitudes frente a la organización, el puesto o el ambiente laboral de desempeño (Fahed-Sreih, 2012).

\section{Dirección Basada en el Control de Gestión y la Asignación de Recompensas}

Según Speklé y Verbeeten (2014), el desempeño organizacional en el sector público depende de los objetivos, de la capacidad de seleccionar indicadores no distorsionado y del grado en que los gerentes conocen y controlan el proceso de transformación, donde uno de los principales componentes de un sistema de control de gestión es la asignación de recompensas, esta asignación de recompensas debe ser realizada de forma mecánica, basado en hechos objetivos y medibles, con cantidades monetarias o incentivos intangibles asignados de acuerdo con el cumplimiento de estos valores objetivos y medibles. Los procesos para recompensar a las personas constituyen los elementos fundamentales para incentivar y motivar a los trabajadores de la organización, siempre que los objetivos organizacionales sean alcanzados y los objetivos individuales sean satisfechos.

\section{Dirección Basada en el Control de Gestión y la Cultura de Mejora Continua}

Un Sistema de Gestión Integrada (SGI), posibilita y simplifica la implantación en un único sistema de gestión, con mayor participación de los trabajadores, alcanzando mayores logros en los objetivos propuestos, aumentando la competitividad de la empresa, mejorando la confianza de los clientes y, en consecuencia, mejorando la imagen y el éxito empresarial (Fraguela et al., 2011). Con el surgimiento de la teoría de la organización, se acentuó la importancia de la información. Una organización es un sistema conformado por personas, recursos materiales e información. Esta última determina el "orden y el caos" entre los individuos, los recursos y en la interrelación personas-recursos. Por esta razón, debe considerarse a las organizaciones como sistemas de información. El impacto de los cambios económicos, políticos, culturales, tecnológicos y otros ha originado una revolución en materia de gestión de información en las organizaciones, se transformaron entonces las normas, los conceptos, los procedimientos, el comportamiento, así como los productos y los servicios, una nueva actitud permea el quehacer cotidiano de la proyección y el desarrollo de las actividades de información; indiscutiblemente el nuevo modelo de gestión tiene como base indispensable la gestión del conocimiento (Aja, 2002).

\section{Dirección Basada en el Control de Gestión y el Logro de Resultados Desafiantes}

Lograr resultados desafiantes, implica alcanzar objetivos complejos y de mayor valor para el cliente y para la organización. Las teorías relativas al control de gestión de la organización han ido avanzando a medida que se han descubierto y considerado nuevas variables que afectan la actuación de la organización y de sus miembros, tanto a nivel interno como externo. Estas también han evolucionado en la medida en la que lo ha hecho la propia realidad de las organizaciones. En efecto, su desarrollo ha estado ligado tanto a los avances de la literatura especializada como a las transformaciones vividas por la sociedad, a los cambios del mercado, a su internacionalización y al incremento de la competitividad (Pérez et al., 2015). La dirección debe llevar sus funciones a la prevención de hechos que generen pérdidas o incidentes costosos a la organización desde el punto de vista financiero y humano, evaluaciones separadas o individuales y la evaluación son actividades de monitoreo que se realizan en forma constante, no como las auditorías periódicas efectuadas por los auditores internos (Vega-de la Cruz y Nieves-Julbe, 2016).

Es poco probable que una organización alcance ventajas competitivas provechosas si la dirección se realiza con base a un sistema de control de la gestión que sea capaz de integrar, desde el nivel estratégico hasta el operativo, la información necesaria para tomar decisiones acertadas y oportunas (Comas et al., 2014). De esta manera, se aprecia que el control de gestión es importante en todo tipo de organizaciones ya que su función principal se orienta a lograr un mayor conocimiento de las tareas que se desarrollan y sus resultados, lo cual, por otra parte, permite de una forma más adecuada el cumplimiento de los objetivos organizacionales como plantea Bossy (2001) "El objetivo del control de gestión es poner en marcha unos sistemas de control, 
formales e informales, que van a hacer posible el desarrollo de las actividades necesarias para alcanzar las metas fijadas a corto y largo plazo" (Peña, 2014).

\section{Incidencia de una Cultura de Mejora Continua en el Cumplimiento de Resultados Desafiantes}

El proceso de mejora de la productividad y calidad es una acción permanente e iterativa de toda la organización, es una actitud que se desarrolla por parte de todo el personal y que permite mantener el interés por la innovación, por la creatividad, por hacer las cosas cada vez mejor y satisfacer en mayor medida las necesidades de los clientes. Si se dispone de una actitud de mejora continua, la empresa tratará siempre de buscar el límite por hacer con unos recursos determinados (Cárdenas y Fecci, 2007). Dado que las certificaciones en calidad son decisiones voluntarias de las empresas, las empresas en estudio, como práctica de mejoramiento de la calidad, usan esquemas locales propios que invitan a que las sugerencias de mejora sean presentadas por trabajadores, siguiendo un enfoque de abajo hacia arriba, es decir, alentando el desarrollo de los empleados, generando oportunidades de crecimiento y recompensando las sugerencias de mejora. De esta manera, los directivos de estas organizaciones consideran que mediante las mejoras graduales presentadas por los mandos bajos y apoyadas por los mandos medios, la gestión de la calidad total podrá alcanzarse en el largo plazo (Alvarado y Pumisacho, 2017).

\section{Incidencia del Desarrollo de Competencias en el Cumplimiento de Resultados}

Según Werther y Davis (2008), la capacitación es un proceso continuo, aun cuando al ingreso de personal nuevo se le dé una inducción adecuada, con frecuencia, es preciso entrenarlos o capacitarlos en las labores para las que fueron contratados y/o proporcionarles nuevos conocimientos necesarios para el desempeño de un puesto, al igual que los empleados con experiencia que son ubicados en nuevos puestos, pueden requerir capacitación para desempeñar adecuadamente su trabajo. Es posible que aún los candidatos internos no posean las habilidades o que también tengan hábitos incorrectos que requieran corregirse. Siempre será necesario mantener un equilibrio entre las aptitudes y actitudes de los trabajadores y los requerimientos del puesto, en los últimos años se tiene un incremento en la necesidad de tener más y mejores conocimientos, el hombre moderno sufre una intensa curiosidad y un indomable afán inquisitivo.

García (2011), sostiene que los beneficios organizacionales más potentes son: incrementar la productividad y la calidad, contribuir a mantener bajos los costos de operación las distintas áreas, contribuir positivamente en el manejo de conflictos y tensiones, y finalmente permite el establecimiento y logro de metas tanto individuales como organizacionales. Las técnicas del desarrollo organizacional por lo general se aplican en una secuencia definida. Las evidencias muestran que los cambios que hacen hincapié en las personas y la organización como un todo son más profundos y eficaces. En el fondo, el desarrollo organizacional representa un verdadero cúmulo de esfuerzos conjuntos para cambiar a la organización por medio del cambio de actitudes y comportamientos de las personas que trabajan en ella, donde el profesionalismo y la diversidad de conocimientos en el puesto de trabajo influirá en el rendimiento (Chiang et al., 2014).

\section{Asignación de Recompensas y el Logro de Resultados Desafiantes}

Los incentivos y beneficios, aunque son motores que promueven la motivación de los colaboradores de una organización y permiten alcanzar las metas propuestas además de brindar a sus miembros las garantías, y la estabilidad esperada. Suele depositarse sobre ellos la responsabilidad de la necesidad de encontrar formas de aumentar el rendimiento de los trabajadores con respecto a las tendencias negativas del desarrollo económico y social (Sierra, 2016). Muchos trabajadores del sector público quieren ayudar a los demás, y a menudo encuentran inmensamente gratificantes las oportunidades para satisfacer sus intenciones altruistas a través de organizaciones públicas. Para apoyar estas afirmaciones, los estudiosos de la motivación del sector público observan que las organizaciones más efectivas encuentran formas únicas de atraer, retener y motivar a los empleados, una estrategia difícil de imitar. El éxito de una organización proviene de administrar a las personas de manera efectiva y brindarles un entorno de trabajo seguro, las mejores oportunidades y caminos para avanzar (Fahed-Sreih, 2012).

\section{Logro de Resultados Desafiantes y la Creación de Valor Público}

El Estado está sujeto a cambios sociales, crisis, tendencias y exigencias de los ciudadanos. Los datos de diferentes encuestas, y en todo América Latina, demuestran que existe cierta desconfianza en los gobiernos, lo que en algunos casos se traduce en un descontento social, que pone constantemente en tela de juicio el cómo el Estado hace su tarea, a quiénes beneficia con sus acciones y cómo lo hace. Surge entonces la necesidad de avanzar hacia la construcción de un Estado rápido y ágil, consciente de que los ciudadanos tienen el derecho de ser parte de las prácticas estatales, de tener políticas públicas acorde a sus necesidades. Todo esto, da como resultado la mejora de la calidad de la función pública junto con la eficiencia en la gestión (Lideres para gobernar, 2017). La producción del valor público necesita no solamente reglas institucionales y una eficiente gestión pública, sino también de factores culturales que permitan fortalecer los contenidos, objetivos y metas de las políticas públicas. Por tanto, la formación de valor público tiene que acreditarse con 
indicadores de medición que aludan a formas de medición sobre cómo se aprovechan los recursos públicos y cómo su aplicación produce mejores externalidades positivas a la sociedad civil. La capacidad pública (Pan Suk, 2007) de producir bienes y servicios no debe ser solamente un ejercicio de generalidad e impersonalidad, sino que se debe conocer cómo se impacta en lo específico la vida de los gobernados y si éstos elevan su calidad de vida, tomando en cuenta la oferta del gobierno a través de un sistema de compromisos públicos que asume.

\section{Modelo Causal}

Considerando las relaciones anteriores, en la figura 1 se muestra el modelo causal propuesto y que busca identificar las variables que influyen en la creación de valor público.

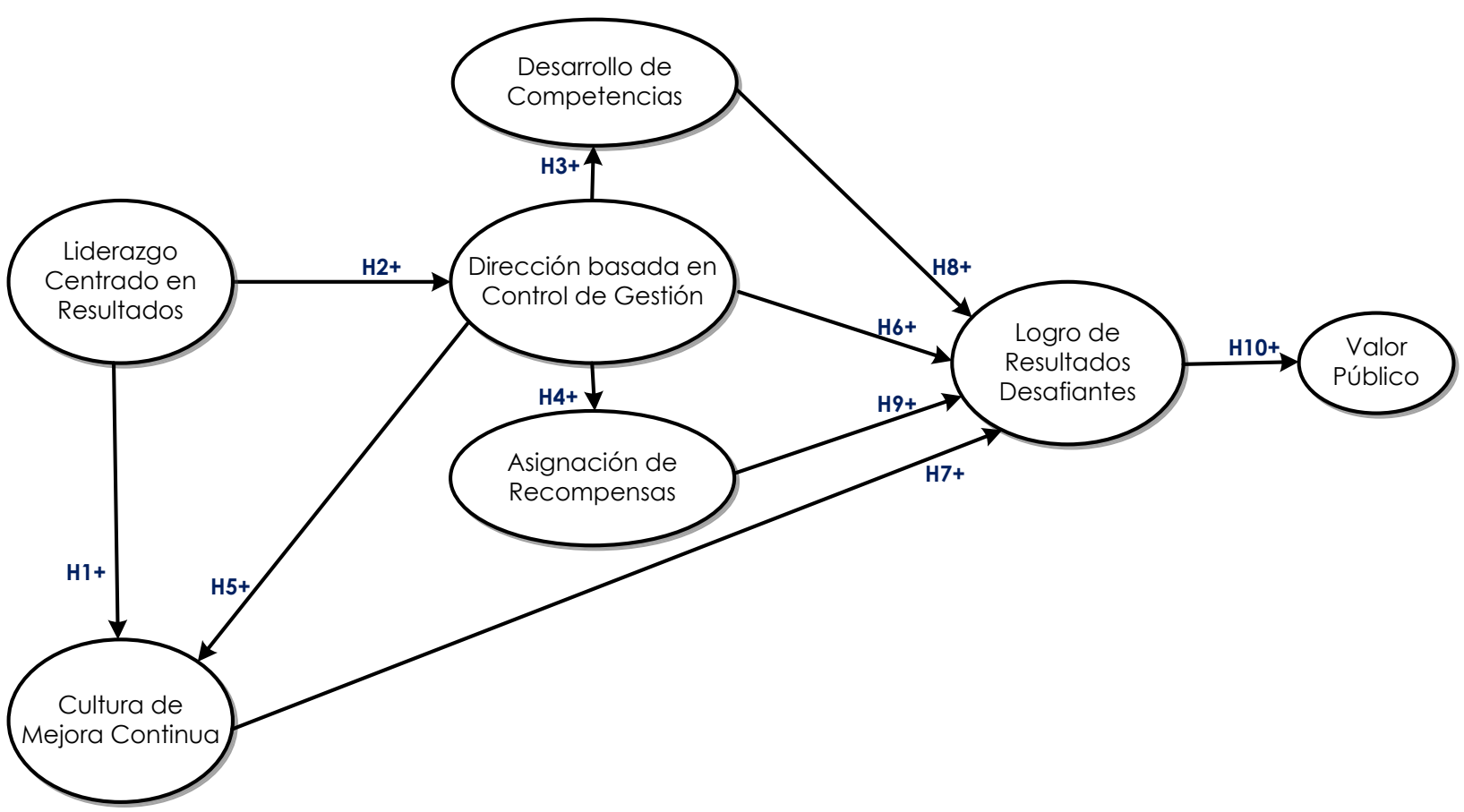

Fig. 1: Modelo Causal Creación de Valor Público.

\section{METODOLOGÍA}

El método empleado para recoger la información es de carácter cuantitativo basado en una encuesta estructurada de tipo Likert, escala de 1 (mínimo valor asociado a respuesta) a 5 (máximo valor asociado a respuesta), el tipo de muestreo es probabilístico con un nivel de confianza del $95 \%$. La encuesta abarco los siguientes ítems: liderazgo basado en resultados; dirección basada en el control de gestión; asignación de recompensas; desarrollo de competencias; logro de resultados desafiantes; cultura de cambio, aprendizaje y mejora continua centrada en el valor de la organización; y la creación de valor público. La investigación fue de carácter exploratorio se apoyó en el software Smart PLS versión 3.3.2, que determinaron si los constructos e indicadores cumplieron con los análisis de fiabilidad y validez, además de determinar las cargas factoriales también llamados indicadores PATH, los que demostraron si las hipótesis efectivamente se aceptan o de lo contrario se rechazan. El trabajo de campo se realizó de manera personal y vía online, en tabla 1 se entregan detalles de la ficha técnica de investigación.

Tabla 1: Ficha técnica de la investigación

\begin{tabular}{|l|l|}
\hline Tipo de muestreo & Muestreo aleatorio \\
\hline Nivel de confianza & $95 \% ; \mathrm{z}=1,96 ; \mathrm{p}=\mathrm{q}=0,5$ (5\% error y $95 \%$ de confiabilidad) \\
\hline Tamaño muestral & 375 funcionarios \\
\hline Unidad de análisis & $\begin{array}{l}\text { Servicios dependientes del Ministerio de Economía, Fomento y } \\
\text { Turismo. }\end{array}$ \\
\hline Método de recogida de la información & Cuestionario presencial y online de 50 preguntas \\
\hline Tipo de pregunta encuesta & $\begin{array}{l}\text { Politómicas tipo Likert (1 a } 5) \text { puntuaciones o categorías y de } \\
\text { selección }\end{array}$ \\
\hline Fecha realización del trabajo de campo & Entre marzo y junio de 2018 \\
\hline
\end{tabular}




\section{Análisis descriptivo}

En tabla 2 y 3 se describen detalles de la muestra que se realiza a 375 funcionarios de los servicios dependientes del Ministerio de Economía, Fomento y Turismo siendo una muestra representativa del 8,58\% de los Servicios dependientes del Ministerio, los encuestados en su mayoría son hombres, representados por el $71,47 \%$ y el $28,53 \%$ restante lo conforma el género femenino, además se divide a la muestra según el uso del CMI, clasificación que fue conformada acorde a la respuesta de los funcionarios. Además, se detallan los porcentajes de encuestados según el tipo de organización o servicio al cual pertenecen, años de servicio, cargo y profesión.

Tabla 2: Análisis descriptivo de la muestra que no utilizan CMI

\begin{tabular}{|c|c|c|c|c|c|}
\hline Variable & Ítem & $\%$ en la Muestra & Variable & Ítem & $\%$ en la Muestra \\
\hline \multirow{2}{*}{ Género } & Hombre & $71,47 \%$ & \multirow{6}{*}{$\begin{array}{l}\text { Año de } \\
\text { Servicio }\end{array}$} & Menos de 1 & $1,07 \%$ \\
\hline & Mujer & $28,53 \%$ & & Entre 1 y 5 & $26,79 \%$ \\
\hline \multirow{16}{*}{ Servicio } & Servicio Nacional de Turismo & \multirow{2}{*}{$9,47 \%$} & & Entre 6 y 10 & $47,86 \%$ \\
\hline & (Sernatur) & & & Entre 11 y 15 & $16,78 \%$ \\
\hline & Servicio Nacional de Pesca & \multirow{2}{*}{$20 \%$} & & Entre 16 y 20 & $7,5 \%$ \\
\hline & (Sernapesca) & & & Más de 20 & $0 \%$ \\
\hline & $\begin{array}{l}\text { Servicio Nacional del } \\
\text { Consumidor (Sernac) }\end{array}$ & $0 \%$ & \multirow{7}{*}{ Cargo } & $\begin{array}{l}\text { Director de } \\
\text { Servicio }\end{array}$ & $0 \%$ \\
\hline & \multirow{2}{*}{$\begin{array}{l}\text { Superintendencia de } \\
\text { Insolvencia y } \\
\text { Reemprendimiento }\end{array}$} & \multirow{2}{*}{$0 \%$} & & Jefe de Servicio & $0 \%$ \\
\hline & & & & Profesional & $55,73 \%$ \\
\hline & \multirow{2}{*}{$\begin{array}{l}\text { Corporación de Fomento de la } \\
\text { Producción (Corfo) }\end{array}$} & \multirow{2}{*}{$44,21 \%$} & & Administrativo & $15,47 \%$ \\
\hline & & & & Fiscalizador & $1,87 \%$ \\
\hline & \multirow{2}{*}{$\begin{array}{l}\text { El Servicio de Cooperación } \\
\text { Técnica (Sercotec) }\end{array}$} & \multirow[b]{2}{*}{$26,31 \%$} & & Técnico & $1,6 \%$ \\
\hline & & & & Auxiliar & $0 \%$ \\
\hline & $\begin{array}{l}\text { Fiscalía Nacional Económica } \\
\text { (FNE) }\end{array}$ & $0 \%$ & \multirow{5}{*}{$\begin{array}{l}\text { Rango de } \\
\text { Edad }\end{array}$} & 18 - 25 años & $5,36 \%$ \\
\hline & $\begin{array}{l}\text { Instituto Nacional de } \\
\text { Estadísticas }\end{array}$ & $0 \%$ & & 26 - 35 años & $36,43 \%$ \\
\hline & $\begin{array}{l}\text { Instituto Nacional de } \\
\text { Propiedad Industrial (Inapi) }\end{array}$ & $0 \%$ & & 36 - 45 años & $40 \%$ \\
\hline & Sistema de Empresas (SEP) & $0 \%$ & & 46 - 55 años & $18,21 \%$ \\
\hline & InvestChile & $0 \%$ & & 56 años o más & $0 \%$ \\
\hline
\end{tabular}

Tabla 3: Análisis descriptivo de la muestra que utilizan CMI

\begin{tabular}{|c|c|c|c|c|c|}
\hline Variable & Ítem & $\%$ en la Muestra & Variable & Ítem & $\%$ en la Muestra \\
\hline \multirow{2}{*}{ Género } & Hombre & $66,32 \%$ & \multirow{6}{*}{$\begin{array}{l}\text { Año de } \\
\text { Servicio }\end{array}$} & Menos de 1 & $1,06 \%$ \\
\hline & Mujer & $33,68 \%$ & & Entre 1 y 5 & $37,89 \%$ \\
\hline \multirow{15}{*}{ Servicio } & Servicio Nacional de Turismo & \multirow{2}{*}{$9,47 \%$} & & Entre 6 y 10 & $44,21 \%$ \\
\hline & (Sernatur) & & & Entre 11 y 15 & $11,58 \%$ \\
\hline & Servicio Nacional de Pesca & \multirow{2}{*}{$20 \%$} & & Entre 16 y 20 & $5,26 \%$ \\
\hline & (Sernapesca) & & & Más de 20 & $0 \%$ \\
\hline & Superintendencia de & \multirow{2}{*}{$0 \%$} & \multirow{6}{*}{ Cargo } & Jefe de Servicio & $1,6 \%$ \\
\hline & Reemprendimiento & & & Profesional & $12,53 \%$ \\
\hline & Corporación de Fomento de la & \multirow{2}{*}{$44,21 \%$} & & Administrativo & $9,34 \%$ \\
\hline & Producción (Corfo) & & & Fiscalizador & $0,8 \%$ \\
\hline & El Servicio de Cooperación & \multirow{2}{*}{$26,31 \%$} & & Técnico & $1,06 \%$ \\
\hline & Técnica (Sercotec) & & & Auxiliar & $0 \%$ \\
\hline & $\begin{array}{l}\text { Fiscalía Nacional Económica } \\
\text { (FNE) }\end{array}$ & $0 \%$ & \multirow{5}{*}{$\begin{array}{l}\text { Rango de } \\
\text { Edad }\end{array}$} & 18 - 25 años & $5,26 \%$ \\
\hline & $\begin{array}{l}\text { Instituto Nacional de } \\
\text { Estadísticas }\end{array}$ & $0 \%$ & & 26 - 35 años & $43,16 \%$ \\
\hline & $\begin{array}{l}\text { Instituto Nacional de } \\
\text { Propiedad Industrial (Inapi) }\end{array}$ & $0 \%$ & & 36 - 45 años & $29,47 \%$ \\
\hline & Sistema de Empresas (SEP) & $0 \%$ & & 46 - 55 años & $22,11 \%$ \\
\hline & InvestChile & $0 \%$ & & 56 años o más & $0 \%$ \\
\hline
\end{tabular}




\section{Fiabilidad individual de los indicadores}

Con la finalidad de evaluar la viabilidad del análisis factorial, se cuantificó el índice Kaiser-Meyer-Olkin (KMO) que para cada uno de los factores fue superior a 0,50 y la prueba de esfericidad de Bartlett (PEB) la que debe ser significativa $(p<0,05)$. Para esto se utilizó el software SPSS Statistics versión 20 utilizando el método de extracción de máxima verosimilitud, en tabla 4 se muestran los resultados observados.

Tabla 4: Índice KMO \& PEB

\begin{tabular}{|l|c|c|}
\hline Constructo & KMO & Significancia de Barlett \\
\hline Liderazgo centrado en resultados (LCR) & 0,789 & 0,000 \\
\hline Dirección basada en Control de Gestión (DBCG) & 0,850 & 0,000 \\
\hline Desarrollo de competencias (DC) & 0,855 & 0,000 \\
\hline Asignación de recompensas (AR) & 0,826 & 0,000 \\
\hline Logro de Resultados desafiantes (LOGR) & 0,688 & 0,000 \\
\hline Cultura de mejora continua (CULMC) & 0,743 & 0,000 \\
\hline Valor público (VP) & 0,635 & 0,000 \\
\hline
\end{tabular}

Por otra parte, para aceptar un indicador como integrante de un constructo se consideró las cargas factoriales con valores superiores a 0,4 sugerido por Hair et al. (2014), no obstante, para obtener más rigurosidad en los datos se eliminaron aquellas cargas factoriales con valores inferiores a 0,5. En tabla 5 se muestran los resultados de las cargas factoriales, las variables LCR6, DBCG1, DBCG2, DBCG3, DBCG5, DBCG7, DBCG8, DBCG9, DBCG12, DBCG16, LOGR1, LOGR2, LOGR3, LOGR8 y CULMC3, no cumplen con el mínimo establecido y por tanto fueron eliminadas.

Tabla 5: Cargas Factoriales

\begin{tabular}{|c|c|c|c|c|c|}
\hline Constructo & Indicador & $\begin{array}{c}\text { Carga } \\
\text { Factorial }(\lambda)\end{array}$ & Constructo & Indicador & $\begin{array}{c}\text { Carga } \\
\text { Factorial }(\lambda)\end{array}$ \\
\hline \multirow{6}{*}{$\begin{array}{l}\text { Liderazgo centrado en } \\
\text { resultados }\end{array}$} & LCR1 & 0,866 & \multirow[t]{5}{*}{ Desarrollo de competencias } & DC1 & 0,851 \\
\hline & LCR2 & 0,756 & & DC2 & 0,835 \\
\hline & LCR3 & 0,857 & & DC3 & 0,776 \\
\hline & LCR4 & 0,860 & & DC4 & 0,849 \\
\hline & LCR5 & 0,869 & & DC5 & 0,783 \\
\hline & LCR6 & $-0,056$ & \multirow[t]{5}{*}{ Asignación de recompensas } & AR1 & 0,920 \\
\hline \multirow{17}{*}{$\begin{array}{l}\text { Dirección basada en Control } \\
\text { de Gestión }\end{array}$} & DBCG1 & 0,213 & & AR2 & 0,836 \\
\hline & DBCG2 & 0,211 & & AR3 & 0,795 \\
\hline & DBCG3 & 0,341 & & AR4 & 0,811 \\
\hline & DBCG4 & 0,508 & & AR5 & 0,682 \\
\hline & DBCG5 & 0,307 & \multirow{8}{*}{$\begin{array}{l}\text { Logro de Resultados } \\
\text { desafiantes }\end{array}$} & LOGR1 & $-0,153$ \\
\hline & DBCG6 & 0,523 & & LOGR2 & $-0,030$ \\
\hline & DBCG7 & 0,127 & & LOGR3 & 0,083 \\
\hline & DBCG8 & 0,483 & & LOGR4 & 0,840 \\
\hline & DBCG9 & 0,317 & & LOGR5 & 0,719 \\
\hline & DBCG10 & 0,804 & & LOGR6 & 0,846 \\
\hline & DBCG11 & 0,836 & & LOGR7 & 0,855 \\
\hline & DBCG12 & 0,193 & & LOGR8 & $-0,002$ \\
\hline & DBCG13 & 0,850 & \multirow[t]{5}{*}{ Cultura de mejora continua } & CULMC1 & 0,867 \\
\hline & DBCG14 & 0,798 & & CULMC2 & 0,567 \\
\hline & DBCG15 & 0,812 & & CULMC3 & 0,394 \\
\hline & DBCG16 & 0,218 & & CULMC4 & 0,819 \\
\hline & DBCG17 & 0,565 & & CULMC5 & 0,852 \\
\hline \multirow{3}{*}{ Valor público } & VP1 & 0,916 & & & \\
\hline & VP2 & 0,833 & & & \\
\hline & VP3 & 0,798 & & & \\
\hline
\end{tabular}




\section{Fiabilidad y Validez del constructo}

Para evaluar la consistencia interna de los indicadores que miden los constructos reflectivos, se analiza la fiabilidad del constructo por medio del alfa de Cronbach y el coeficiente de fiabilidad compuesta (IFC). El Alfa de Cronbach permite estimar la fiabilidad de un instrumento de medida a través de un conjunto de ítems que se espera que midan el mismo constructo o dimensión teórica. La validez de un instrumento se refiere al grado en que el instrumento mide aquello que pretende medir. En la Tabla 6 se muestra que el Alfa de Cronbach presenta una alta consistencia interna en los 7 constructos analizados, ya que supera los niveles recomendados de 0,7 (George y Mallery, 2003). De la misma forma, el índice de fiabilidad compuesta (IFC) de un constructo permite medir la consistencia interna del bloque de indicadores, es decir, las variables observables del constructo. En la tabla 6 se observa que el IFC supera los valores recomendados de 0,7 . Por lo tanto, se verifica la consistencia interna de las variables del instrumento de evaluación.

\section{Validez Convergente}

La validez convergente se comprueba a través de la varianza extraída media (AVE) de los constructos. En la tabla 6 , el coeficiente AVE para todos los valores alcanzados superan el valor mínimo recomendado de 0,5 y son significativos al $95 \%$, se confirma que las variables explican más del $50 \%$ de la varianza de sus constructos y por lo tanto existe validez convergente (Esposito et al., 2010).

Tabla 6: Índice de Fiabilidad Interno, Índice de Fiabilidad Compuesta y Validez Convergente

\begin{tabular}{|l|c|c|c|}
\hline Constructo & $\begin{array}{c}\text { Alfa de } \\
\text { Cronbach }\end{array}$ & IFC & $\begin{array}{c}\text { Validez Convergente } \\
\text { (AVE) }\end{array}$ \\
\hline Liderazgo centrado en resultados (LCR) & 0,897 & 0,897 & 0,710 \\
\hline Dirección basada en Control de Gestión (DBCG) & 0,933 & 0,876 & 0,541 \\
\hline Desarrollo de competencias (DC) & 0,875 & 0,873 & 0,671 \\
\hline Asignación de recompensas (AR) & 0,864 & 0,865 & 0,660 \\
\hline Logro de Resultados desafiantes (LOGR) & 0,834 & 0,873 & 0,667 \\
\hline Cultura de mejora continua (CULMC) & 0,797 & 0,826 & 0,617 \\
\hline Valor Público (VP) & 0,807 & 0,850 & 0,723 \\
\hline
\end{tabular}

\section{Validez Discriminante}

Los valores de la matriz de correlaciones entre constructos que está formada por la raíz cuadrada del coeficiente AVE debe ser superior al resto de su misma columna (Espejel et al., 2013). Al respecto, se indica un procedimiento inverso en donde se calcula la raíz cuadrada de la AVE, que ha de ser mayor que las correlaciones que presentan con el resto de constructos, los resultados expuestos en tabla 7 son: LCR: 0,735; DBCG: 0,843; DC: 0,819; AR: 0,812; LOGR: 0,817; CULMC: 0,786 y VP: 0,850, lo que corresponde a indicadores que garantizan la validez discriminante del modelo propuesto.

Tabla 7: Validez Discriminante

\begin{tabular}{|c|c|c|c|c|c|c|c|}
\hline Constructo & AR & CULMC & DC & DBCG & LCR & LOGR & VP \\
\hline AR & 0,812 & & & & & & \\
\hline CULMC & 0,055 & 0,786 & & & & & \\
\hline DC & 0,545 & 0,032 & 0,819 & & & & \\
\hline DBCG & 0,168 & 0,230 & 0,114 & 0,843 & & & \\
\hline LCR & 0,205 & 0,241 & 0,077 & 0,159 & 0,735 & & \\
\hline LOGR & 0,163 & 0,639 & 0,345 & 0,375 & 0,225 & 0,817 & \\
\hline VP & 0,065 & 0,709 & 0,042 & 0,141 & 0,182 & 0,325 & 0,850 \\
\hline
\end{tabular}

\section{RESULTADOS}

Se planteó el método de modelos estructurales a través de los Mínimos Cuadrados Parciales (Partial Least Squares, PLS), debido a que este método está orientado a modelos de carácter predictivo y exploratorio, en donde el conocimiento teórico no está del todo desarrollado, como es el caso el modelo propuesto que es un modelo propio con recolección de algunas teorías. Para los análisis se utilizó el software SmartPLS. 
Se evalúan las relaciones causales que se proponen en el modelo, por medio de la varianza explicada $\left(R^{2}\right)$ (Johnson y Kuby, 2011). La varianza explicada de las variables endógenas o dependientes debe ser igual o mayor que 0,1 (Falk y Miller, 1992). Como se aprecia en tabla 8, todos los constructos cumplen con este criterio. Se puede apreciar como el valor $\mathrm{R}^{2}$ para el Valor Público es de 0,475 , lo que quiere decir que el $47,5 \%$ de la varianza de este constructo es explicada por el modelo, lo que según Henseler et al. (2009) modelos con $\mathrm{R}^{2}$ sobre 0,33 se consideran moderados. En otras palabras, pendientes de contrastar la validez de estas relaciones, lo cual se hará seguidamente, se puede afirmar que aproximadamente el $48 \%$ de la varianza del Valor Público viene determinada por el logro de resultados desafiantes.

\section{Índice de Bondad de Ajuste (GoF)}

Esposito et al. (2010) sugiere un criterio global de bondad de ajuste para los modelos estructurales PLS. Estos autores proponen que el índice de bondad de ajuste global se dé por medio de la raíz cuadrada de la multiplicación de la media aritmética del análisis de la varianza extraída (AVE) y la media aritmética de la varianza explicada $\left(R^{2}\right)$ de las variables endógenas o dependientes. Como se observa en tabla 8, el índice de bondad de ajuste (GoF) es 0,404 demostrando que se tiene un buen ajuste en el modelo de medida y en el modelo estructural, cumpliendo así con el criterio empírico de que la medida de bondad de ajuste debe variar entre 0 y 1, a mayor valor, mejor será el índice (Tenenhaus y Esposito 2005).

Tabla 8: Varianza Explicada e Índice de bondad de ajuste

\begin{tabular}{|l|c|c|}
\hline Constructo & $\begin{array}{c}\text { Validez Convergente } \\
\text { (AVE) }\end{array}$ & $\mathrm{R}^{2}$ \\
\hline Liderazgo centrado en resultados (LCR) & 0,710 & \\
\hline Dirección basada en Control de Gestión (DBCG) & 0,541 & 0,125 \\
\hline Desarrollo de competencias (DC) & 0,671 & 0,112 \\
\hline Asignación de recompensas (AR) & 0,660 & 0,103 \\
\hline Logro de Resultados desafiantes (LOGR) & 0,667 & 0,519 \\
\hline Cultura de mejora continua (CULMC) & 0,617 & 0,163 \\
\hline Valor Público (VP) & 0,723 & 0,475 \\
\hline \multicolumn{1}{|c|}{0,404} \\
\hline
\end{tabular}

\section{Contraste de Hipótesis}

Con respecto a los coeficientes PATH o pesos de regresión estandarizados ( $\beta$ ), los valores deseables deberían estar por encima de 0,3 siendo 0,2 un valor mínimo aceptable (Chin, 1998). Como se observa en tabla 9 las hipótesis H3, H4, H5 y H9 no superan el valor de 0,2 que es el mínimo valor aceptado, las demás relaciones cumplen con la regla de estar sobre el mínimo valor aceptado.

Tabla 9: Contraste de hipótesis

\begin{tabular}{|c|l|c|c|}
\hline Hipótesis & Relaciones & Coeficiente PATH & Contraste \\
\hline H1 & LCR $\rightarrow$ CULMC & 0,248 & ACEPTA \\
\hline H2 & LCR $\rightarrow$ DBCG & 0,275 & ACEPTA \\
\hline H3 & DBCG $\rightarrow$ DC & 0,114 & RECHAZA \\
\hline H4 & DBCG $\rightarrow$ AR & 0,168 & RECHAZA \\
\hline H5 & DBCG $\rightarrow$ CULMC & 0,035 & RECHAZA \\
\hline H6 & DBCG $\rightarrow$ LOGR & 0,265 & ACEPTA \\
\hline H7 & CULMC $\rightarrow$ LOGR & 0,680 & ACEPTA \\
\hline H8 & DC $\rightarrow$ LOGR & 0,222 & ACEPTA \\
\hline H9 & AR $\rightarrow$ LOGR & 0,126 & RECHAZA \\
\hline$H 10$ & LOGR $\rightarrow$ VP & 0,325 & ACEPTA \\
\hline
\end{tabular}




\section{DISCUSIÓN}

El Liderazgo centrado en Resultados incide positiva y directamente en la Dirección basada en el control de gestión y Cultura de mejora continua del servicio, explicándolas en un $27,5 \%$ y 24,8\%, respectivamente. Así se evidencia que en los procesos de servicios públicos, se debe influir y movilizar a las personas cotidianamente hacia el logro de resultados desafiantes, a través del control de gestión, como también disponer de un ambiente de trabajo donde exista una cultura de mejora continua y por ende desarrollo de competencias, posibilitando que entre los diferentes procesos de servicio público se genere un alineamiento vertical y horizontal, que facilite el trabajo colaborativo, centrado en metas consensuadas por los stakeholders participantes, pues así finalmente se creará valor público, escenario imprescindible estratégicamente en Chile, ya que la competitividad del país tiene como un factor restrictivo la imagen del sector público.

Considerando que el Liderazgo centrado en resultados tiene una influencia directa y positiva en la Dirección basada en el control de gestión y esta última en conjunto con el Desarrollo de Competencias y la Cultura de Mejora Continua, influyen directa y positivamente con significancia estadística, en el Logro de Resultados Desafiantes de esta organización pública, en un $26,5 \%, 22,2 \%$ y $68 \%$ respectivamente, que a su vez influye de forma directa y significativa en el Valor Público del Ministerio, explicándolo en un 32,5\%. Entonces, una gestión sistémica de estas variables promoverá la creación de valor público, generando parcialmente un "ecosistema innovador", donde la colaboración entre distintos actores (Comunidad, Ministerio y Gobierno), crean un contexto que favorece el surgimiento y sostenibilidad de nuevos servicios y modelos de trabajo, donde el control de gestión como herramienta que apoya a la dirección de las organizaciones, a través de la información generada periódicamente, permitirá a los decisores públicos conocer y aprender de forma proactiva, según lo que suceda en cada momento, en los procesos considerados críticos para la organización.

Se puede afirmar con evidencias empíricas significativas, que para esta organización del Estado, la Dirección basada en el control de gestión no tiene influencia estadística en la Asignación de recompensas (16,8\%), Desarrollo de competencias $(11,4 \%)$ y Cultura de mejora continua (3,5\%). Es decir, una Dirección basada en Control de Gestión, no puede producir los impactos teóricos especificados en el modelo planteado, por si sola resulta insuficiente, debe ser un trabajo sistémico de la organización. Actualmente en el Gobierno de Chile, existe un marco regulatorio y normas del ministerio, determinadas por la Dirección de Presupuesto (DIPRES), unidad dependiente del Ministerio de Hacienda, que restringen la influencia de la Dirección basada en Resultados. Si bien una Dirección Basada en Resultados, puede justificar objetiva y técnicamente a través de la evaluación, que ciertos cargos merecen mejor recompensa que otros, las asignaciones de estas recompensas están determinadas según los lineamientos de la DIPRES. Por lo cual los funcionarios no perciben las recompensas recibidas como merecidas o justas, influyendo en su desempeño diario. A su vez, instalar una Cultura de Mejora continua es un desafío a largo plazo, la mejora continua en las personas no se puede lograr solamente a través de tener un Sistema de Dirección de Control de Gestión se requiere de otros estilos de gestión que deben ser parte de la organización, por ejemplo, para que efectivamente la Cultura de Mejora Continua se instale, deben estar asociados las recompensas a los logros y eso aún no ocurre. El Sector Público en Chile no se caracteriza por tener como base central de la promoción vertical o el desarrollo dentro de la organización a la meritocracia, sino más bien prima la amistocracia y las relaciones políticas, dañando la profesionalización en la gestión de los Servicios Públicos impidiendo la consolidación de la Cultura de Mejora Continua.

Complementariamente, hay escenario donde los recursos asociados a recompensas y programas de desarrollo de competencias, son inferiores a la demanda interna, por lo cual las recomendaciones técnicas que los responsables de procesos puedan realizar fundamentados en sus informes de control de gestión, generalmente no se consideran. Así el Liderazgo centrado en resultados en esta organización pública, tiene un foco más de control, menos sistémico y menos orientado a lograr alineamientos organizacionales que potencien la sinergia. Se conecta más a los objetivos que desea alcanzar el Gobierno, en desmedro de los objetivos personales de su capital humano, de allí entonces que el potencial del control de gestión se subutiliza, dañando así el compromiso organizacional. Lo anterior es relevante, si consideramos que un Liderazgo centrado en resultados, implica motivar, comunicar, enseñar y empoderar (poder de tomar decisiones con autonomía) a su gente, tomando como insumo los resultados que se generan de procesos de control de gestión, donde la evaluación de los programas de acción (en el largo plazo representan las estrategias de desarrollo país), requiere de un ecosistema que estimule evolucionar desde el aprendizaje en los diagnósticos participativos en cada proceso, a la toma de decisiones que aporten valor a la comunidad interna y a la sociedad.

En este contexto, es una responsabilidad moral que los programas públicos del Ministerio de Economía, Fomento y Turismo, generen continuamente valor a la sociedad, por ello sus directivos tienen el gran desafío de transformar el escenario actual y así lograr que sus Liderazgos centrados en resultados comprometidos 
públicamente en sus programas del gobierno, se traduzcan y se operativicen en una Dirección basada en control de gestión, que influya en el desarrollo de competencias, la asignación de recompensas y la instalación de una cultura de mejora continua. Así los funcionarios públicos tendrán la convicción que los programas de acción y estrategias de desarrollo país, como sus resultados, también son beneficiosos para ellos, en consecuencia, la seguirán empoderadamente y ejecutarán cotidianamente, aumentando los Logros de resultados desafiantes que impactan directamente en el Valor público generado.

\section{CONCLUSIONES}

Se concluye que el Liderazgo centrado en resultados tiene una influencia directa y positiva en la Dirección basada en el control de gestión y en conjunto con el Desarrollo de Competencias y la Cultura de Mejora Continua, influyen directa y positivamente en el Logro de Resultados Desafiantes y que esta última explica significativamente el Valor Público generado por los servicios dependientes del Ministerio de Economía, Fomento y Turismo de Chile.

El Valor Público en un servicio, se crea al interior de la institución, debido a la gestión diaria de su capital humano, a través del Liderazgo basado en Resultados, la Dirección centrada en Control de Gestión y el Desarrollo de Competencias, permitiendo lograr Resultados Desafiantes, que contribuyen directamente al logro de la misión institucional y con ello a la misión ministerial, promoviendo el desarrollo sostenible y sustentable del país.

El valor moderado de explicación del Valor Público de los servicios dependientes del Ministerio de Economía, Fomento y Turismo, deja al descubierto que se deben incorporar nuevas variables para lograr una mayor explicación del modelo, estas pueden ser: la calidad de servicio, las políticas del gobierno nacional e internacional, los impactos de la globalización, entre otras.

La gestión sistémica de las variables causales del modelo, promoverá la creación de valor público, donde el control de gestión permitirá a los decisores públicos conocer y aprender de forma proactiva, según lo que suceda en cada momento, en los procesos considerados críticos para la organización.

\section{AGRADECIMIENTOS}

Los autores agradecen el apoyo entregado por el Ministerio de Economía, Fomento y Turismo de Chile y a la Universidad de La Serena, para terminar exitosamente esta investigación.

\section{REFERENCIAS}

Aguilar, L., Gobernanza y gestión pública, Fondo de cultura económica, México DF, (2015)

Aja, L., Gestión de información, gestión del conocimiento y gestión de la calidad en las organizaciones, ACIMED, 10(5), 7-8, (2002)

Alvarado, K. y Pumisacho, V., Prácticas de mejora continua, con enfoque Kaizen, en empresas del distrito metropolitano de Quito: Un estudio exploratorio, Revista Capital intangible, Universidad politécnica de Cataluña, 13(2), 479-497, (2017)

Anthony, R. y Govindarajan, V., Management Control Systems, 12ª ed., McGraw-Hill / Irwin, Boston-Massachusetts, United States of America, (2007)

Arboleda, J. y Arias, L., Uso de análisis multicriterio para medir la efectividad de los procesos estratégicos en empresas de servicios públicos, Colección académica de ciencias sociales, 3(1), 67-82, (2016)

Barney, J., Gaining and sustaining competitive advantage, 4ํㅜ ed., Pearson, Upper Saddle River, N.J., (2011)

Bossy, M., Control de Gestión, MassonBrasilia: Ed. Ática S.A. Ceac, Barcelona, España, (2001)

Cárdenas, L. y Fecci, E., Propuesta de un modelo de gestión para PYMES, centrado en la mejora continua, Síntesis Tecnológica, 3(2), 59-67, (2007)

Chakravarthy, B., Measuring strategic performance, https://doi.org/10.1002/smj.4250070505, Strategic Management Journal, 7(5), 437-458, (1986)

Chiang, M.M., Gómez, N.M. y Salazar, C.M., Satisfacción laboral y estilos de liderazgo en instituciones públicas y privadas de educación de Chile, Cuadernos de Administración, 30 (52), 65-74, (2014)

Chiang, M.M. y San Martín, N.J., Análisis de la satisfacción y el desempeño laboral en los funcionarios de la Municipalidad de Talcahuano, https://doi.org/10.4067/S0718-24492015000300001, Ciencia \& trabajo, 17(54), 159-165, (2015)

Chin, W.W., The Partial Least Squares Approach to Structural Equation Modeling, In G. A., Marcoulides [ed.], Modern Methods for Business Research, Mahwah, NJ: Lawrence Erlbaum Associates, 295-336, (1998) 
Comas-Rodríguez, R., Nogueira-Rivera, D. y Medina-León, A., El control de gestión y los sistemas de información: propuesta de herramientas de apoyo, Ingeniería Industrial, 35(2), 214-228, (2014)

Espejel, J.E., Camarena, D.M. y Sandoval, S.A., Alimentos tradicionales en sonora, México: factores que influyen en su consumo, https://doi.org/10.15446/innovar.v24n53.43920, Innovar: Revista de Ciencias Administrativas y Sociales, 24(53), 127-139, (2013)

Esposito, V., Chin, W., Henseler, J. y Wang, H., Handbook of partial leastsquares: Concepts, Methods and applications, $1^{\text {st }}$ edition, Berlin: Springer-Verlag Berlin Heidelberg, (2010)

Fahed-Sreih, J., The Effect of Investing in Hiring, Human Resource Planning and Employee Development on Labor Productivity: Case of Lebanon, Journal of International Business Research, 11(1), 29-51, (2012)

Falk, R.F. y Miller, N.B., A primer for soft modeling, 1ra edition, Akron, O H: University of Akron Press, (1992)

Fraguela, J.A., Carral, L., Iglesias, G., Castro, A. y Rodríguez, M.J., La integración de los sistemas de gestión necesidad de una nueva cultura empresarial, DYNA, 78(167), 44-49, (2011)

Gamboa, J., Puente, S. y Vera, P., Importancia del control interno en la política, Revista Publicando, 3(8), 487-502, (2016)

García, J.M., El proceso de capacitación, sus etapas e implementación para mejorar el desempeño del recurso humano en las organizaciones, Contribuciones a la economía, ISSN: 1696-8360, En línea: http://www.eumed.net/ce/2011b/jmgl.html, 1-18, (2011)

George, D. y Mallery, P., SPSS for windows step by step: A simple guide and reference, $4^{\text {a }}$ ed., Allyn y Bacon, Boston, United States of America, (2003)

Hair, J.F., Black, W.C., Babin, B.J. y Anderson, R.E., Multivariate data analysis, $7^{a} \underline{a}$ ed., Pearson, United States of America, (2014).

Henseler, J., Ringle, C.M. y Sinkovics, R.R., The use of partial least squares path modeling in international marketing, https://doi.org/10.1108/S1474-7979(2009)0000020014, Advances in International Marketing, 20, 277-319, (2009)

Johnson, R.R. y Kuby, P.J., Elementary statistics, 11ª ed., Cengage Learning, (2011)

Kaplan, R. y Norton, D., Mapas estratégicos: Convirtiendo los activos intangibles en resultados tangibles, Ediciones Gestión 2000, Barcelona, España, (2004)

Líderes para gobernar, El estado debe generar valor público, Recuperado el 20 de enero de 2018 de: http://lideresparagobernar.org/valor-publico/html, (2017)

Marín-García, J.A., Bautista-Poveda, Y. y García-Sabater, J.J., Etapas en la evolución de la mejora continua: Estudio multicaso, Intangible Capital, 10(3), 584-618, (2014)

Pan Suk, K., Desafíos a la capacidad pública en la era de una administración pública en evolución y reforma de gobierno, Gestión y Política Pública, 16(2), 511-537, (2007)

Peña, F., Propuesta de un sistema de control de gestión para las contralorías municipales del estado Carabobo, Maestría en ciencias contables, Universidad de Carabobo, Venezuela, (2014)

Pérez, A.R., Vázquez, A.W. y Levín, S., El control de gestión y talento humano: Conceptos y enfoques, https://doi.org/10.12804/rev.univ.empresa.29.2015.01, Universidad \& Empresa, 17(29), 13-33, (2015)

Porporato, M. y García, N., Sistemas de control de gestión: un estudio exploratorio de su efecto sobre el desempeño organizacional, Revista Latinoamericana de Administración, 47, 61-77, (2011)

Prado, J.C., The Implementation of Continuous Improvement Through the Participation of Personnel: A Case Study, Production and Inventory Management Journal, 39(2): 11-15, (1998)

Prieto, B. y Santidrián, A., Control de gestión y creación de valor a través de los sistemas de medición del rendimiento, Documento $\mathrm{N}^{\circ} 40$ de la serie de principios de contabilidad de gestión AECA, 1aㅡ ed., Universidad de Burgos, (2016)

Rivero, K. y Galarza, J., El cuadro de mando integral como una alternativa para el seguimiento y control de estrategias en las instituciones de educación superior, Revista cubana de educación superior, 36(3), 85-95, (2017)

Robbins, S., y Judge, T., Comportamiento organizacional, 15ª ed., Pearson, México, (2015)

Schroeder, R.G., Bates, K.A. y Junttila, M.A., A Resource-Based View of Manufacturing Strategy and the Relationship to Manufacturing Performance, https://doi.org/10.1002/smj.213, Strategic Management, 23(2), 105-117, (2002)

Sierra, E., Análisis de las ventajas y desventajas de las estrategias de incentivos, compensación y beneficios y su impacto en las organizaciones, Universidad Militar Nueva Granada, Seminario de investigación, (2016)

Speklé, R.F. y Verbeeten, F.H.M., The use of performance measurement systems in the public sector: Effects on performance, https://doi.org/10.1016/j.mar.2013.07.004, Management Accounting Research, 25(2), 131-146, (2014) 
Tello, P., Bastidas, D. y Pisconte, J., Programa de formación: Desarrollo de capacidades para el fortalecimiento de las organizaciones políticas. Gestión Pública, Institute for Democracy and Electoral Assistance, (2009)

Tenenhaus, M. y Esposito, V., PLS regression, PLS path modeling and generalized Procrustean analysis: a combined approach for multiblock analysis, https://doi.org/10.1002/cem.917, Journal of Chemometrics, 19, 145-153, (2005)

Toro-Alava, W.J., Carranza-Quimi, W.D., Espinoza-Mendoza J.E. y Malave-Galarza L.A., Sistemas de control de gestión, una herramienta administrativa para las decisiones gerenciales, Revista administración y finanzas, 4(10), 38-49, (2007)

Ulrich, D. y Smallwood, N., Building a leadership brand, Harvard Business Review, 85(7-8), 92-100, (2007)

Varela, F., Liderazgo en el Sector Público. Aproximaciones y Desencuentros con el Sector Privado, Julio 2012, Administración Pública, Universidad de Santiago de Chile, 1-12, (2012)

Vega-de la Cruz, L.O. y Nieves-Julbe, A.F., Procedimiento para la gestión y supervisión y monitoreo de control interno, Ciencias Holguín, 22(1), 1-19, (2016)

Werther, W. y Davis, K., Administración de recursos humanos. El capital humano de las empresas, 6ª ed., McGraw-Hill, México DF, (2008) 